\title{
MAGNETIC PROPERTIES OF THE DOPED TWO-DIMENSIONAL ANTIFERROMAGNET
}

\author{
A. SHERMAN \\ Institute of Physics, Riia 142, 51014 Tartu, Estonia \\ M. SCHREIBER \\ Institut für Physik, Technische Universität, D-09107 Chemnitz \\ and School of Engineering and Science, International University Bremen \\ Campus Ring 1, D-28759 Bremen, Federal Republic of Germany

\begin{abstract}
The variety of the normal-state magnetic properties of cuprate high- $T_{c}$ superconductors is interpreted based on the self-consistent solution of the self-energy equations for the two-dimensional $t$ - $J$ model. The observed variations of the spin correlation length with the hole concentration $x$, of the spin susceptibility with $x$ and temperature $T$ and the scaling of the static uniform susceptibility are well reproduced by the calculated results. The nonmonotonic temperature dependence of the $\mathrm{Cu}$ spin-lattice relaxation rate is connected with two competing tendencies spin gap and the growth of the susceptibility in this frequency region with the temperature broadening of the maximum in the susceptibility.
\end{abstract} \\ in the low-frequency susceptibility: its temperature decrease due to the increasing
}

Magnetic properties of cuprate perovskites have been extensively studied during the last years, both because of their unusual behavior and in the hope that they might provide insight into the physical origin of high- $T_{c}$ superconductivity. These properties provide a good test for models proposed for the description of cuprates and approximations used for their solution. One of the most popular models is the two-dimensional $t-J$ model of $\mathrm{Cu}-\mathrm{O}$ planes. This mpdel describes the low-energy dynamics of the realistic three-band Hubbard model. 6 Recently we have suggested an approximate method for the solution of the $t$ - $J$ model, which has merits of retaining the rotation symmetry of spin components in the paramagnetic state and of the absence of any predefined magnetic ordering. The obtained magnetic properties of the model appear to be close to those observed in the cuprate perovskites which allows us to clarify mechanisms leading to the unusual behavior.

The Hamiltonian of the two-dimensional $t-J$ model reads

$$
H=\sum_{\mathbf{n} \mathbf{m} \sigma} t_{\mathbf{n m}} a_{\mathbf{n} \sigma}^{\dagger} a_{\mathbf{m} \sigma}+\frac{1}{2} \sum_{\mathbf{n m}} J_{\mathbf{n m}}\left(s_{\mathbf{n}}^{z} s_{\mathbf{m}}^{z}+s_{\mathbf{n}}^{+1} s_{\mathbf{m}}^{-1}\right)-\mu \sum_{\mathbf{n}} X_{\mathbf{n}},
$$

where $a_{\mathbf{n} \sigma}=|\mathbf{n} \sigma\rangle\langle\mathbf{n} 0|$ is the hole annihilation operator, $\mathbf{n}$ and $\mathbf{m}$ label sites of the square lattice, $\sigma= \pm 1$ is the spin projection, $|\mathbf{n} \sigma\rangle$ and $|\mathbf{n} 0\rangle$ are site states corresponding to the absence and presence of a hole on the site. For nearest neighbor interactions $t_{\mathbf{n m}}=t \sum_{\mathbf{a}} \delta_{\mathbf{n}, \mathbf{m}+\mathbf{a}}$ and $J_{\mathbf{n m}}=J \sum_{\mathbf{a}} \delta_{\mathbf{n}, \mathbf{m}+\mathbf{a}}$ where $t$ and $J$ are the hopping and exchange constants and the four vectors a connect nearest neighbor sites. The spin- $\frac{1}{2}$ operators can be written as $s_{\mathbf{n}}^{z}=\frac{1}{2} \sum_{\sigma} \sigma|\mathbf{n} \sigma\rangle\langle\mathbf{n} \sigma|$ and $s_{\mathbf{n}}^{\sigma}=$ $|\mathbf{n} \sigma\rangle\langle\mathbf{n},-\sigma|, \mu$ is the chemical potential and $X_{\mathbf{n}}=|\mathbf{n} 0\rangle\langle\mathbf{n} 0|$.

The method suggested in Ref. 7 is based on Mori's projection operator techniquel which allows one to represent Green's functions in the form of continued fractions and gives a way for calculating their elements. The residual term

mpda: submitted to World Scientific on November 13, 2018 
of the fraction can be approximated by the decoupling which reduces this manyparticle Green's function to a product of simpler functions. In this way we obtained the following self-energy equations for the hole $G(\mathbf{k} t)=-i \theta(t)\left\langle\left\{a_{\mathbf{k} \sigma}(t), a_{\mathbf{k} \sigma}^{\dagger}\right\}\right\rangle$ and spin $D(\mathbf{k} t)=-i \theta(t)\left\langle\left[s_{\mathbf{k}}^{z}(t), s_{-\mathbf{k}}^{z}\right]\right\rangle$ Green's functions:

$$
\begin{aligned}
& D(\mathbf{k} \omega)=\frac{\left[4 J \alpha\left(\Delta+1+\gamma_{\mathbf{k}}\right)\right]^{-1} \Pi(\mathbf{k} \omega)+4 J C_{1}\left(\gamma_{\mathbf{k}}-1\right)}{\omega^{2}-\Pi(\mathbf{k} \omega)-\omega_{\mathbf{k}}^{2}}, \\
& G(\mathbf{k} \omega)=\phi\left[\omega-\varepsilon_{\mathbf{k}}+\mu^{\prime}-\Sigma(\mathbf{k} \omega)\right]^{-1},
\end{aligned}
$$

where the self-energies $\Pi(\mathbf{k} \omega)$ and $\Sigma(\mathbf{k} \omega)$ read

$$
\begin{aligned}
\operatorname{Im} \Pi(\mathbf{k} \omega)= & \frac{16 \pi t^{2} J}{N}\left(\Delta+1+\gamma_{\mathbf{k}}\right) \sum_{\mathbf{k}^{\prime}}\left(\gamma_{\mathbf{k}}-\gamma_{\mathbf{k}+\mathbf{k}^{\prime}}\right)^{2} \int_{-\infty}^{\infty} d \omega^{\prime}\left[n_{F}\left(\omega+\omega^{\prime}\right)-n_{F}\left(\omega^{\prime}\right)\right] \\
& \times A\left(\mathbf{k}+\mathbf{k}^{\prime}, \omega+\omega^{\prime}\right) A\left(\mathbf{k}^{\prime} \omega^{\prime}\right), \\
\operatorname{Im} \Sigma(\mathbf{k} \omega)= & \frac{16 \pi t^{2}}{N \phi} \sum_{\mathbf{k}^{\prime}} \int_{-\infty}^{\infty} d \omega^{\prime}\left[\gamma_{\mathbf{k}-\mathbf{k}^{\prime}}+\gamma_{\mathbf{k}}+\operatorname{sgn}\left(\omega^{\prime}\right)\left(\gamma_{\mathbf{k}-\mathbf{k}^{\prime}}-\gamma_{\mathbf{k}}\right){\sqrt{\frac{1+\gamma_{\mathbf{k}^{\prime}}}{1-\gamma_{\mathbf{k}^{\prime}}}}}^{2}\right. \\
& \times\left[n_{B}\left(-\omega^{\prime}\right)+n_{F}\left(\omega-\omega^{\prime}\right)\right] A\left(\mathbf{k}-\mathbf{k}^{\prime}, \omega-\omega^{\prime}\right) B\left(\mathbf{k}^{\prime} \omega^{\prime}\right) .
\end{aligned}
$$

In the above formulas $\gamma_{\mathbf{k}}=\frac{1}{4} \sum_{\mathbf{a}} \exp (i \mathbf{k a}), n_{F}(\omega)=[\exp (\omega / T)+1]^{-1}, n_{B}(\omega)=$ $[\exp (\omega / T)-1]^{-1}, A(\mathbf{k} \omega)=-\pi^{-1} \operatorname{Im} G(\mathbf{k} \omega)$ and $B(\mathbf{k} \omega)=-\pi^{-1} \operatorname{Im} D(\mathbf{k} \omega)$ are the hole and spin spectral functions, $\mu^{\prime}=\mu-4 F_{1} \phi^{-1} t+3 C_{1} \phi^{-1} J, \phi=\frac{1}{2}(1+x)$ and

$$
\omega_{\mathbf{k}}^{2}=16 J^{2} \alpha\left|C_{1}\right|\left(1-\gamma_{\mathbf{k}}\right)\left(\Delta+1+\gamma_{\mathbf{k}}\right), \quad \varepsilon_{\mathbf{k}}=\left(4 \phi t+6 C_{1} \phi^{-1} t-3 F_{1} \phi^{-1} J\right) \gamma_{\mathbf{k}},
$$

The hole concentration $x$, the spin $C_{1}=\left\langle s_{1}^{+1} s_{\mathbf{1 + a}}^{-1}\right\rangle$ and hole $F_{1}=\left\langle a_{1}^{\dagger} a_{\mathbf{l}+\mathbf{a}}\right\rangle$ nearest neighbor correlations are determined by the relations

$$
\begin{aligned}
& x=N^{-1} \sum_{\mathbf{k}} \int_{-\infty}^{\infty} d \omega n_{F}(\omega) A(\mathbf{k} \omega), \quad C_{1}=\frac{2}{N} \sum_{\mathbf{k}} \gamma_{\mathbf{k}} \int_{0}^{\infty} d \omega \operatorname{coth}\left(\frac{\omega}{2 T}\right) B(\mathbf{k} \omega), \\
& F_{1}=N^{-1} \sum_{\mathbf{k}} \gamma_{\mathbf{k}} \int_{-\infty}^{\infty} d \omega n_{F}(\omega) A(\mathbf{k} \omega) .
\end{aligned}
$$

The parameter of vertex correction $\alpha$ is set equal to its value in the undoped case, with introduced artificial broadening $\alpha=1.802$. As known, $\theta_{\text {in }}$ the considered two-dimensional system the long-range antiferromagnetic ordering is destroyed at any nonzero $T$ and, as can be seen from the above formulas, at any nonzero $x$. The arising state with short-range order has to satisfy the constraint of zero site magnetization $\left\langle s_{1}^{z}\right\rangle=0$ which can be written in the form

$$
\frac{1}{2}(1-x)=\frac{2}{N} \sum_{\mathbf{k}} \int_{0}^{\infty} d \omega \operatorname{coth}\left(\frac{\omega}{2 T}\right) B(\mathbf{k} \omega) .
$$

This equation fixes the parameter $\Delta$ describing the spin gap at $(\pi, \pi)$.

The same derivation applied to the transversal spin Green's function $\left\langle\left\langle s_{\mathbf{k}}^{-1} \mid s_{\mathbf{k}}^{+1}\right\rangle\right\rangle$ gives $\left\langle\left\langle s_{\mathbf{k}}^{-1} \mid s_{\mathbf{k}}^{+1}\right\rangle\right\rangle=2 D(\mathbf{k} \omega)$ indicating that the approximation used retains properly the rotation symmetry of spin components in the paramagnetic state.

mpda: submitted to World Scientific on November 13, 2018 
Figure 1. The dispersion of spin excitations. Vertical bars show decay widths $|\operatorname{Im} \Pi(\mathbf{k} \omega)| /\left(2 \omega_{\mathbf{k}}\right)$. Points $Y, M$ and $S$ corresponds to $\mathbf{k}=(0, \pi),(\pi, \pi)$ and $(\pi / 2, \pi / 2)$, respectively.

Figure 2. (a) Spin correlations vs. $x$ for $T=0.02 t$. (b) Spin correlations along the diagonal [i.e., $\mathbf{l}=(m, m)]$ of the crystal for $x=0.12$. The respective temperatures are indicated near the curves.

For low $x$ and $T$ the magnitude of the dispersion $\varepsilon_{\mathbf{k}}$ is small in comparison with $t$ manifesting the band narrowing in the antiferromagnetic surrounding.

Equations (2)-(6) form a closed set which can be solved by iteration. We carried out such calculations for a $20 \times 20$ lattice and the parameters $t=0.5 \mathrm{eV}, J=0.1 \mathrm{eV}$ corresponding to cuprates. Small artificial broadenings $-\eta$ and $-2 \eta \omega_{\mathbf{k}}, \eta=0.02 t$, were added to $\operatorname{Im} \Sigma(\mathbf{k} \omega)$ and $\operatorname{Im} \Pi(\mathbf{k} \omega)$, respectively, to widen narrow lines and to stabilize the iteration procedure.

The frequencies of spin excitations satisfy the equation

$$
\omega^{2}-\operatorname{Re}(\mathbf{k} \omega)-\omega_{\mathbf{k}}^{2}=0
$$

[see Eq. (2)]. For low $x$ and $T$ their dispersion is close the dispersion of spin waves (see Fig. 1a). The main difference is the spin gap at $(\pi, \pi)$ the magnitude of which grows with $x$ and $T$ (Fig. 1 $\mathrm{b}$ ). In an infinite crystal this gap is directly connected with the spin correlation length $\xi$. Indeed, for large distances and low $T$ we find

$$
\left\langle s_{\mathbf{1}}^{z} s_{\mathbf{0}}^{z}\right\rangle=N^{-1} \sum_{\mathbf{k}} \mathrm{e}^{i \mathbf{k} \mathbf{l}} \int_{0}^{\infty} d \omega \operatorname{coth}\left(\frac{\omega}{2 T}\right) B(\mathbf{k} \omega) \propto \mathrm{e}^{i \mathbf{Q 1}}(\xi /|\mathbf{1}|)^{1 / 2} \mathrm{e}^{-|\mathbf{l}| / \xi},
$$

where $\mathbf{Q}=(\pi, \pi)$ and $\xi=a /(2 \sqrt{\Delta})$ with the intersite distance $a$. For low $x$ we found that $\Delta \approx 0.2 x$ and consequently $\xi \approx a / \sqrt{\Delta}$. This relation has been experimentally observed 10 in $\mathrm{La}_{2-x} \mathrm{Sr}_{x} \mathrm{CuO}_{4}$.

As seen from Fig. 1 $1 \mathrm{~b}$, with growing $x$ the spin excitation branch is destroyed in some region around the $\Gamma$ point - Eq. (7) has no solution for real $\omega$ due to negative $\operatorname{Re} \Pi(\mathbf{k} \omega)$. For fixed $T$ the size of this region grows with $x$. However, as follows from Fig. 1 $1 \mathrm{~b}$, with increasing $T$ the branch is recovered in this region. This result is the consequence of the temperature broadening of the spin-polaron peak in $A(\mathbf{k} \omega)$. Large negative values of $\operatorname{Re} \Pi(\mathbf{k} \omega)$ which are the reason for the lack of a solution of Eq. (7) are connected with this peak. With its broadening $|\operatorname{Re} \Pi|$ becomes smaller.

The spin correlations $C_{m l}=\left\langle s_{\mathbf{1}}^{z} s_{\mathbf{0}}^{z}\right\rangle, \mathbf{l}=(n, m)$, Eq. (8), are shown in Fig. 2. Distant correlations decrease rapidly with $x$. For large enough $x$ and $T$ the correlations decay exponentially with distance in the considered finite lattice.

The magnetic susceptibility is connected with the spin Green's function (2) by the relation $\chi^{z}(\mathbf{k} \omega)=-4 \mu_{B}^{2} D(\mathbf{k} \omega)$, where $\mu_{B}$ is the Bohr magneton. Experiments on inelastic neutron scattering give information on the dynamic spin susceptibility which can be directly compared with the calculated results. Such comparison is carried out in Fig. 3. $\mathrm{YBa}_{2} \mathrm{Cu}_{3} \mathrm{O}_{7-y}$ is a bilayer crystal and the symmetry allows one to divide the susceptibility into odd and even parts. For the antiferromagnetic intrabilayer coupling the odd part of the susceptibility can be compared with the

mpda: submitted to World Scientific on November 13, 2018 
Figure 3. The imaginary part of the spin susceptibility for $\mathbf{k}=(\pi, \pi)$. Curves demonstrate results of our calculations for $T=0.02 t$, and $x=0.043,0.08$ and 0.12 (from top to ppttom). Squares show the imaginary part of the odd spin susceptibility for $\mathbf{k}=(\pi, \pi)$ measured 5 in normal state $\mathrm{YBa}_{2} \mathrm{Cu}_{3} \mathrm{O}_{7-y}$ at $T=100 \mathrm{~K}$ for $y=0.5,0.17$ and 0.03 (from top to bottom).

Figure 4. The uniform static spin susceptibility vs. $T$ (a) and $x$ (b).

calculated results. The oxygen deficiencies $y=0.5,0.17$ and 0.03 in the experimental data in Fig. 3 correspond to the hole concentrations $x=0.07,0.13$ and 0.17 respectively.11 We scale the experimental $\operatorname{Im} \chi$ so that their maximal values coincide approximately with the calculated maxima. In absolute units the calculated maximal values are approximately 2-2.5 times larger than the experimental ones. As seen from Fig. 3, the calculated data reproduce correctly the frequency dependence of the susceptibility, the values of the frequency for which $\operatorname{Im} \chi(\pi, \pi)$ reaches maximum and their evolution with doping. We notice also that the calculated temperature variation of the susceptibility is in good agreement with experiment.

The calculated dependencies of the uniform static spin susceptibility $\chi_{0}=$ $\chi(\mathbf{k} \rightarrow 0, \omega=0)$ on $T$ and $x$ are shown in Fig. 1. In this figure $\chi_{0} / \mu_{B}^{2}$ lies in the range 2-2.6 $\mathrm{eV}^{-1}$ which is close to the values $1.9-2.6 \mathrm{eV}^{-1}$ obtained $\mathrm{t}$ for $\mathrm{YBa}_{2} \mathrm{Cu}_{3} \mathrm{O}_{7-y}$. The temperature dependence of $\chi_{0}$ has a maximum and its temperature $T_{m}$ grows with decreasing $x$. Analogous behavior of $\chi_{0}$ was observed 13 in cuprates for large enough $x$. In Fig. $4 \mathrm{a} T_{m} \approx 600 \mathrm{~K}$ which is close to $T_{m}$ observed 1 in $\mathrm{La}_{2-x} \mathrm{Sr}_{x} \mathrm{CuO}_{4}$ for comparable $x$. As known, in the undoped antiferromagnet $T_{m} \approx J$. On the high-temperature side from the maximum $\chi_{0}(T)$ tends to the Curie-Weiss dependence 1/T. As seen from Fig. 4a, the two curves for the different $x$ are very close in shape and can be superposed by scaling to the same maximal value of $\chi_{\mathrm{f}}$ and $T_{m}$. Analogous scaling was observed 1 in $\mathrm{La}_{2-x} \mathrm{Sr}_{x} \mathrm{CuO}_{4}$. As in experiment, 1 the dependence $\chi_{0}(x)$ in Fig. $4 \mathrm{~b}$ has a maximum. However, the experimental maximum is achieved at $x \approx 0.25$.

It is of interest to clarify the reason for the nonmonotonic behavior of the dependence $\chi_{0}(T)$. From Eqs. (2) and (3) it is clear that $\chi(\mathbf{k}, \omega=0)$ is continuous for small $\mathbf{k}$. Therefore let us consider a small but finite value of $\mathbf{k}$ for which $\chi(\mathbf{k}, 0) \propto \int_{-\infty}^{\infty} d \omega^{\prime} B\left(\mathbf{k} \omega^{\prime}\right) / \omega^{\prime}$. The maximum in $B\left(\mathbf{k} \omega^{\prime}\right)$ is shifted to lower frequencies and its intensity decreases with increasing temperature. However this dependence is superimposed with the function $1 / \omega^{\prime}$ in the integral. Nonmonotonic behavior of $\chi_{0}(T)$ is the consequence of the existence of some optimal location of the maximum in $B\left(\mathbf{k} \omega^{\prime}\right)$ when the area under the curve $B\left(\mathbf{k} \omega^{\prime}\right) / \omega^{\prime}$ is maximal. Johnston's scaling 1 means that the temperature softening of the long-wavelength maximum occurs similarly for different $x$ and that holes and temperature fluctuations act in a similar manner as sources of the softening.

The spin-lattice relaxation and spin-echo decay rates were calculated with the

mpda: submitted to World Scientific on November 13, 2018 
Figure 5. The temperature dependencies of the spin-lattice relaxation and spin-echo decay rates. Open circles with right axes represent experimental results, filled circles with left axes are our calculation (a,c,d) calculations for $\mathbf{H} \| \mathbf{c}$ and $x=0.12$, measurements $(x \approx 0.1) .11$ (b) calculations for nonoriented configuration with $x=0.043$, measurements 2 in $\mathrm{La}_{1.96} \mathrm{Sr}_{0.04} \mathrm{CuO}_{4}$.

use of the equationst

$$
\begin{aligned}
& \frac{1}{{ }^{\alpha} T_{1 \beta} T}=\frac{1}{2 \mu_{B}^{2} N} \sum_{\mathbf{k}}{ }^{\alpha} F_{\beta}(\mathbf{k}) \frac{\operatorname{Im} \chi(\mathbf{k} \omega)}{\omega}, \quad \omega \rightarrow 0 \\
& \frac{1}{{ }^{63} T_{2 G}^{2}}=\frac{0.69}{128 \mu_{B}^{4}}\left\{\frac{1}{N} \sum_{\mathbf{k}}{ }^{63} F_{e}^{2}(\mathbf{k})[\operatorname{Re} \chi(\mathbf{k} 0)]^{2}-\left[\frac{1}{N} \sum_{\mathbf{k}}{ }^{63} F_{e}(\mathbf{k}) \operatorname{Re} \chi(\mathbf{k} 0)\right]^{2}\right\}
\end{aligned}
$$

where the form factors ${ }^{\alpha} F_{\beta}(\mathbf{k})$ and hyperfine coupling constants were taken from Ref. 4. The superscript $\alpha=63$ or 17 indicates that the respective quantity belongs to $\mathrm{Cu}$ and $\mathrm{O}$, respectively. The subscripts $\beta=\|$ or $\perp$ refer to the direction of the applied static magnetic field $\mathbf{H}$ with respect to the axis $\mathbf{c}$ perpendicular to the $\mathrm{Cu}-\mathrm{O}$ plane. The form factor ${ }^{63} F_{e}$ is the filter for the $\mathrm{Cu}$ spin-echo decay time ${ }^{63} T_{2 G}$. Our calculated results and the respective experimental data are given in Fig. 5. The calculations reproduce satisfactorily main peculiarities of the temperature dependence of the spin-lattice and spin-echo decay rates. The growth of $\left({ }^{63} T_{1} T\right)^{-1}$ with decreasing $x$ is connected with the increase of the spectral intensity of spin excitations near $(\pi, \pi)$. For the same $x\left({ }^{63} T_{1} T\right)^{-1}$ is one to two orders of magnitude larger than $\left({ }^{17} T_{1} T\right)^{-1}$. This is a consequence of the spin susceptibility which is strongly peaked near $(\pi, \pi)$ and the form factors which test different $\mathbf{k}$ regions.

In Fig. 5 the calculated values of the spin-lattice relaxation rates are nearly an order of magnitude smaller than the experimental ones. This difference is connected with the approximation made in the calculation of $D(\mathbf{k} \omega)$ the continued fraction of which was cut short in the second link. This leads to an additional power of $\omega$ in the denominator of $B(\mathbf{k} \omega)$.

It is of interest to clarify the reason for the nonmonotonic temperature dependence of $\left({ }^{63} T_{1} T\right)^{-1}$ for moderate $x$ (see Fig. 5a). The main contribution to $\left({ }^{63} T_{1} T\right)^{-1}$ is given by the vicinity of the $(\pi, \pi)$ point. For low $\omega$ in this region $\operatorname{Im} \chi(\mathbf{k} \omega)$ first grows then decreases with increasing $T$. This is connected with two competing tendencies in the spin spectral function: i) the spin gap $\Delta$ grows with $T$ which leads to a decrease of $B$ in the mentioned regions of $\mathbf{k}$ and $\omega$ and ii) the temperature broadening of the maximum in $B$ which leads to its growth in these regions. For moderate $x$ and low $T$ practically $\Delta$ does not depend on $T$, the second tendency dominates and $\left({ }^{63} T_{1} T\right)^{-1}$ grows with $T$. For higher $T$ the gap grows more rapidly with $T$ leading to the predominance of the first tendency and $\left({ }^{63} T_{1} T\right)^{-1}$ decreases with $T$. For small $x$ temperature fluctuations dominate, $\Delta$ grows noticeably even for small $T$ and $\left({ }^{63} T_{1} T\right)^{-1}$ decreases monotonously with $T$ (see Fig. $5 \mathrm{~b}$ ). Due to the form factor ${ }^{17} F_{\|}(\mathbf{k})$, the central part of the Brillouin zone makes the main contribution to $\left({ }^{17} T_{1} T\right)^{-1}$. For moderate $T$ and $x$ the existence of the spin gap affects $B(\mathbf{k} \omega)$ weakly here. For small $\omega$ the temperature dependence of $B(\mathbf{k} \omega)$

mpda: submitted to World Scientific on November 13, 2018 
is determined by broadening. As a consequence, $\left({ }^{17} T_{1} T\right)^{-1}$ grows monotonously with $T$ (see Fig. 同c). The low-temperature decreases of $\left({ }^{63} T_{1} T\right)^{-1}$ and $\chi^{0}$ were considered as manifestations of the spin gap. As follows from the above discussion, these peculiarities are not directly connected with the gap.

This work was partially supported by the ESF grant No. 4022 and by DFG (SFB 393).

\section{References}

1. D. C. Johnston, Phys. Rev. Lett. 62, 957 (1989).

2. T. Imai et al., Phys. Rev. Lett. 70, 1002 (1993).

3. M. Takigawa et al., Phys. Rev. B 43, 247 (1991); 49, 4158 (1994)

4. V. Barzykin and D. Pines, Phys. Rev. B 52, 13585 (1995).

5. H. F. Fong et al., Phys. Rev. B 61, 14773 (2000).

6. A. Sherman and M. Schreiber, Physica C 303, 257 (1998).

7. A. Sherman and M. Schreiber, Phys. Rev. B 65, 134520 (2002).

8. H. Mori, Progr. Theor. Phys. 34, 399 (1965); A. V. Sherman, J. Phys. A 20, 569 (1987).

9. N. D. Mermin and H. Wagner, Phys. Rev. Lett. 17, 1133 (1966).

10. B. Keimer et al., Phys. Rev. B 46, 14034 (1992).

11. J. L. Tallon et al., Phys. Rev. B 51, 12911 (1995).

mpda: submitted to World Scientific on November 13, 2018 


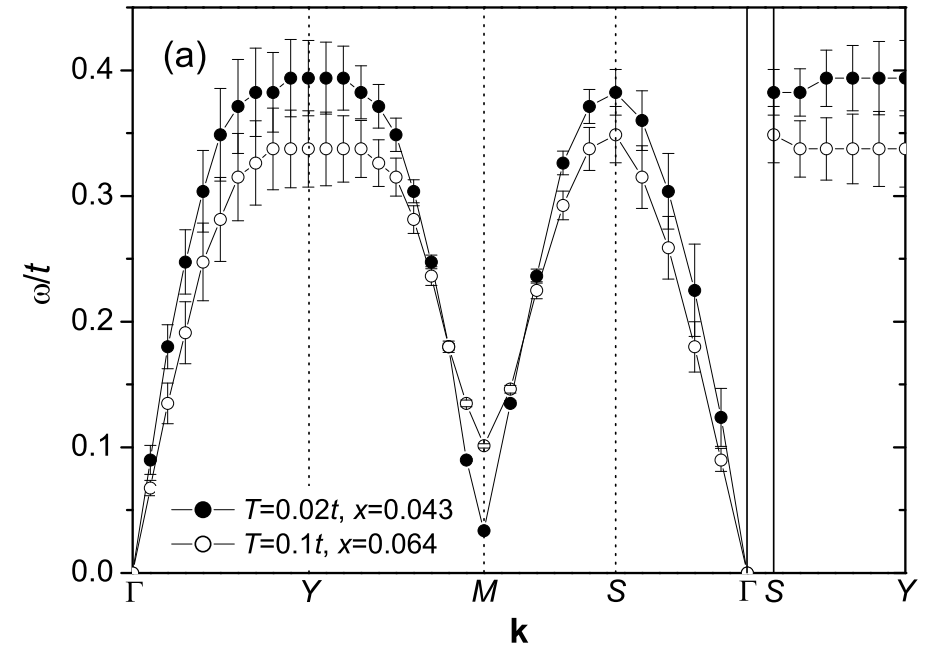




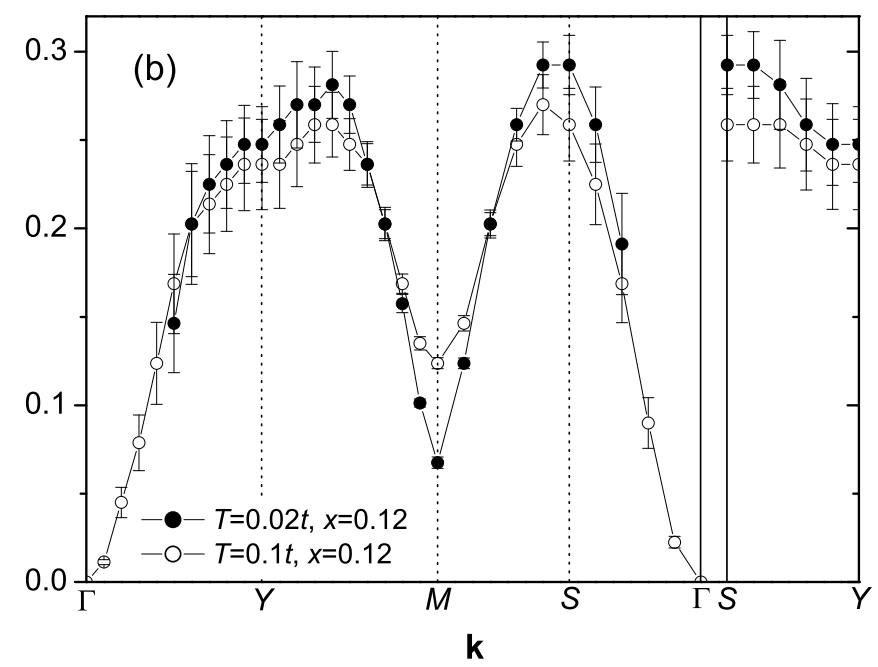




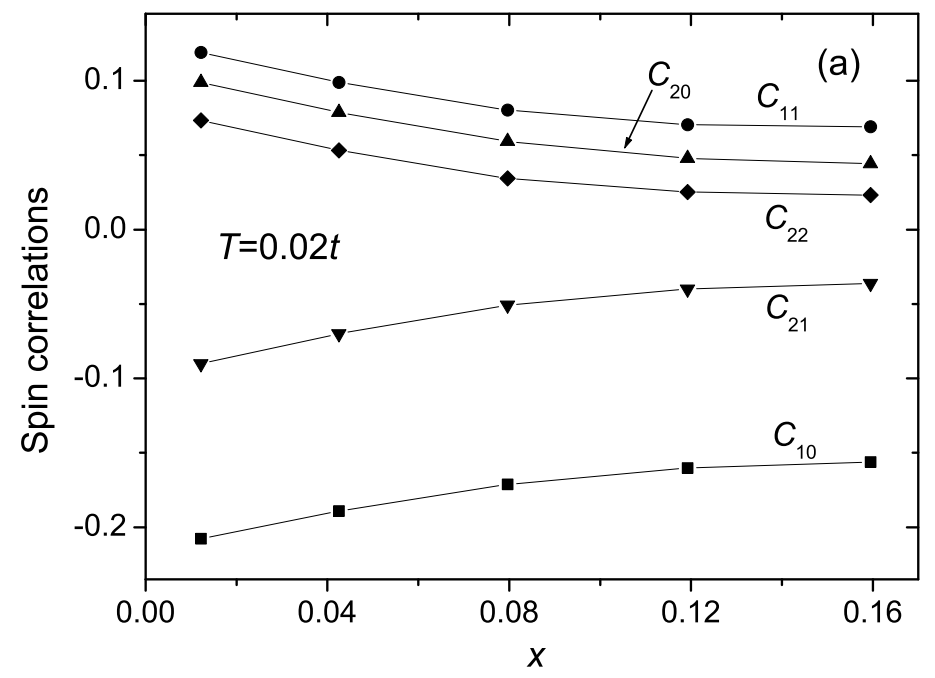




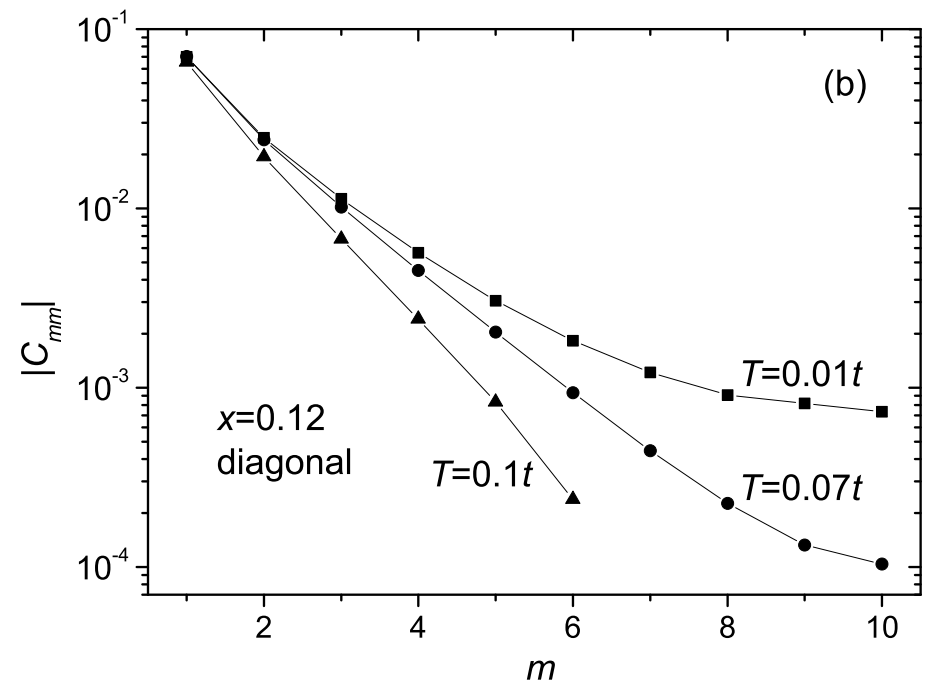




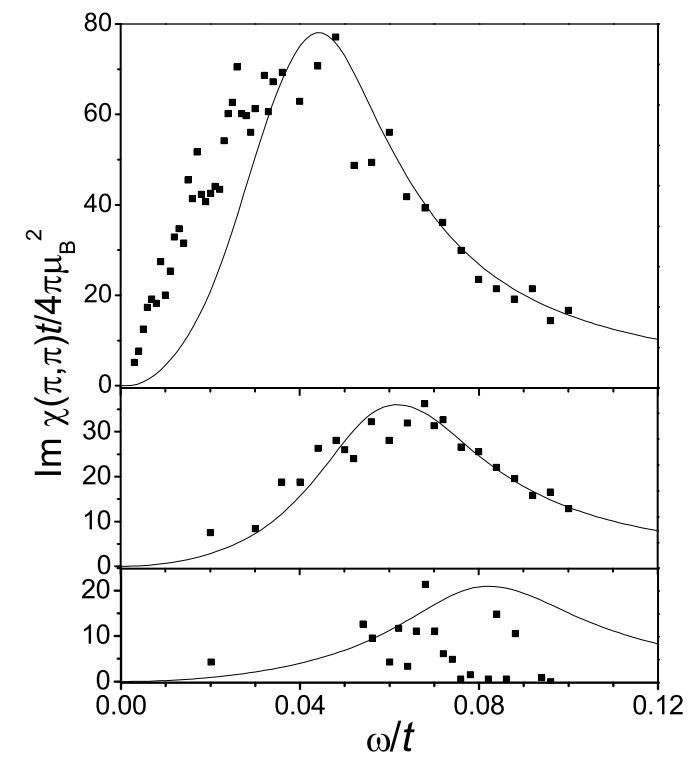




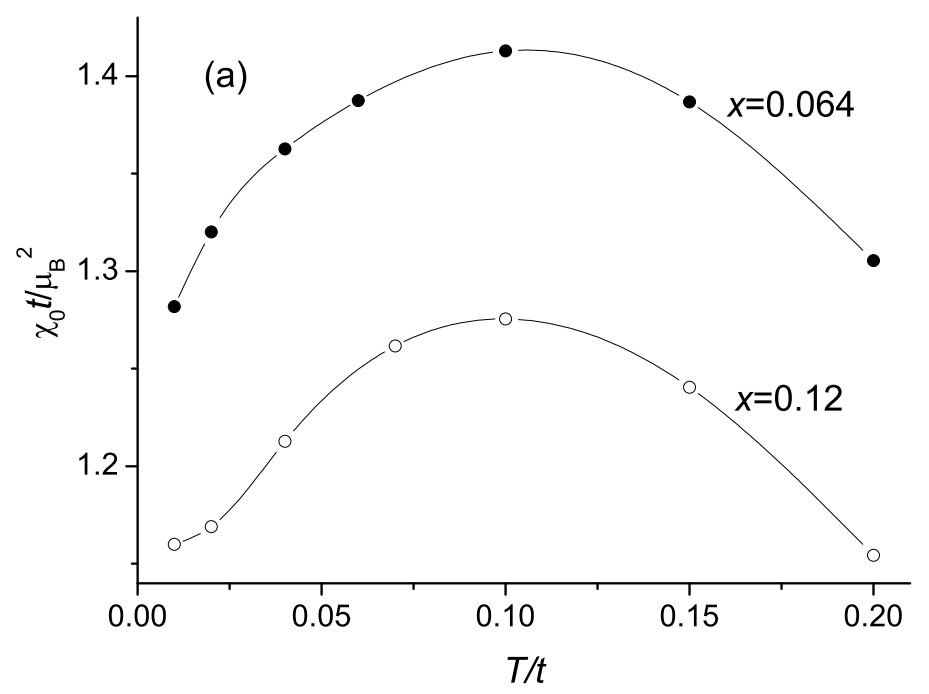




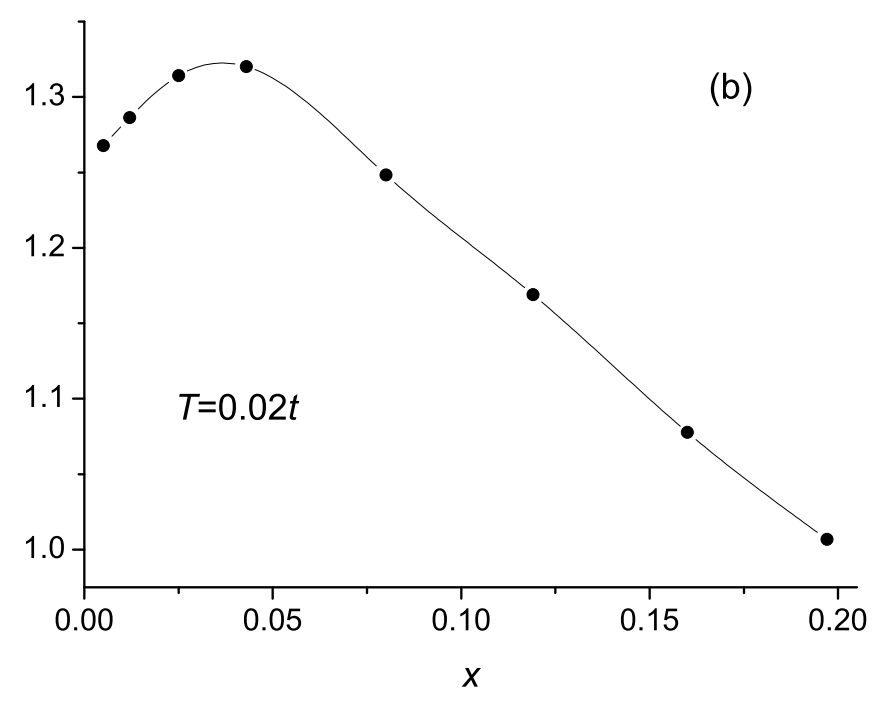




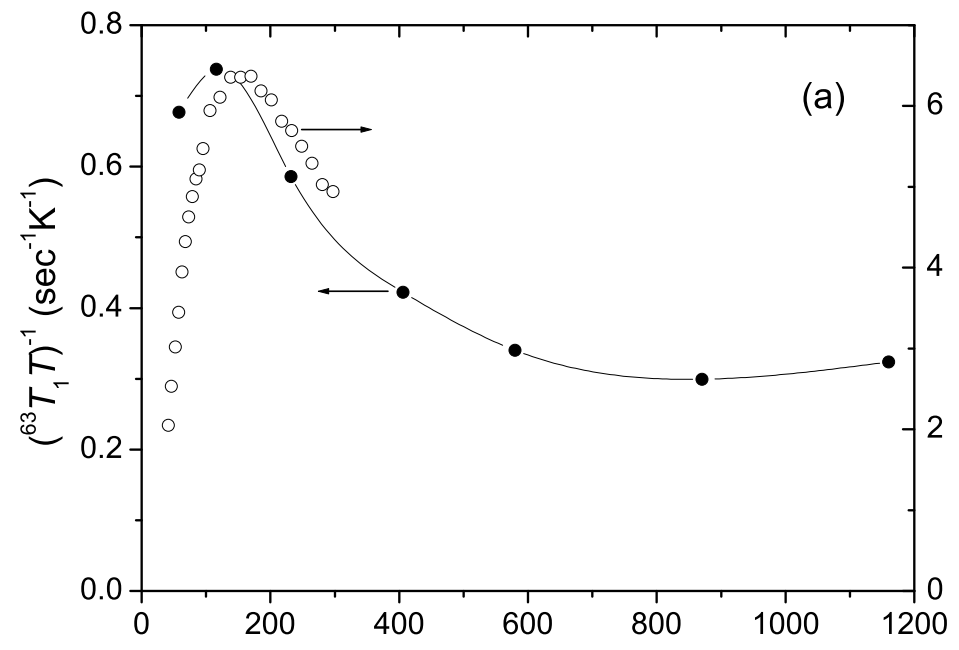




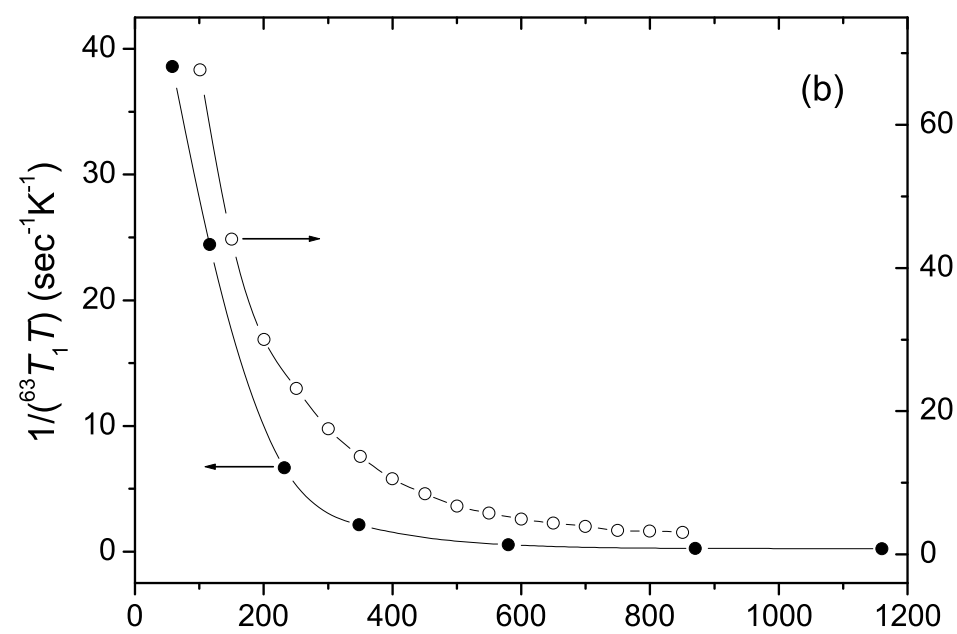




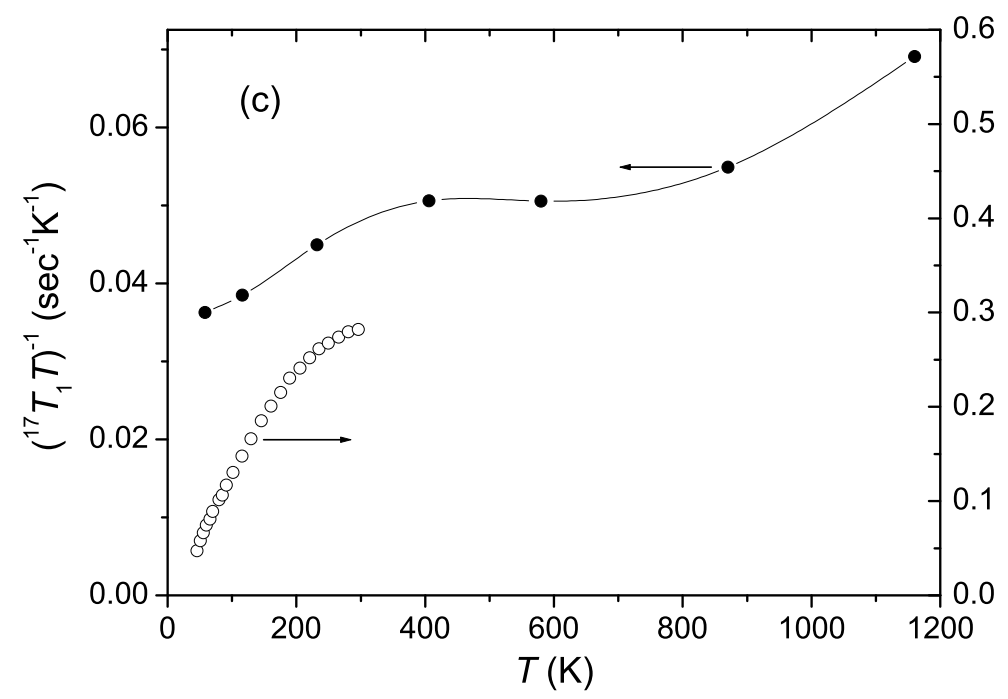




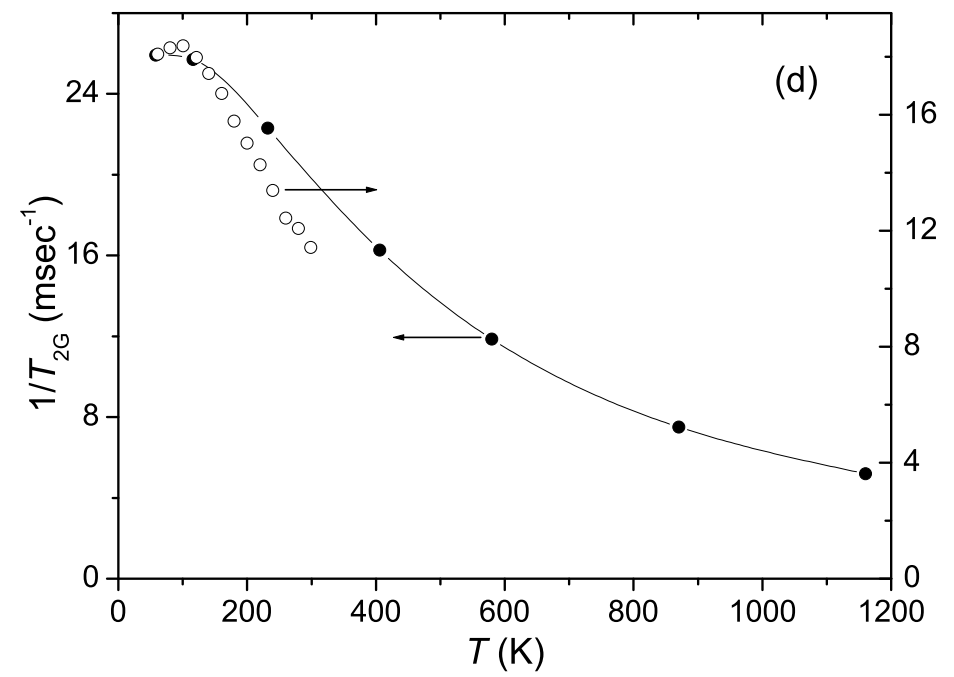

